\title{
The temperature and size effect on the electrical resistivity of $\mathrm{Cu} / \mathrm{V}$
}

\section{multilayer films}

\author{
P.P. Wang ${ }^{\text {a }}$, X.J. Wang ${ }^{\text {b }}$, J.L. Du ${ }^{\text {a }}$, F. Ren ${ }^{\text {c }}$, Y. Zhang ${ }^{\text {d }}$, X. Zhang ${ }^{\text {e }}$, E.G. Fu ${ }^{\text {a, * }}$ \\ ${ }^{a}$ State Key Laboratory of Nuclear Physics and Technology, School of Physics, Peking \\ University, Beijing 100871, P. R. China \\ ${ }^{\mathrm{b}}$ State Key Laboratory of Advanced Optical Communication Systems and Networks, \\ Peking University, Beijing 100871, P.R. China \\ ${ }^{c}$ School of Physics and Technology, Wuhan University, Wuhan, 430072, P.R. China \\ ${ }^{\mathrm{d}}$ Institute of Condensed Matter and Material Physics, School of Physics, Peking University, \\ Beijing 100871, P. R. China \\ ${ }^{\mathrm{e}}$ School of Materials Engineering, Purdue University, West Lafayette, Indianan, 47907, \\ USA
}

*Corresponding author. E-mail address: efu@pku.edu.cn or fuengang@gmail.com (E.G. $\mathrm{Fu})$

\begin{abstract}
The electrical resistivity of sputter-deposited $\mathrm{Cu} / \mathrm{V}$ multilayer films with different individual layer thicknesses varying from 2.5 to $100 \mathrm{~nm}$ was evaluated in the temperature range of $150-300 \mathrm{~K}$. The temperature coefficient of resistivity (TCR) of $\mathrm{Cu} / \mathrm{V}$ multilayer was compared based on the semi-classical theory of Dimmich model. A new model has been proposed to describe the relationship between the resistivity and the individual layer thickness of metallic multilayer film by considering both interface scattering and grain boundary scattering based on Fuchs-Sondheimer method and Mayadas-Shatzkes method.
\end{abstract}

Keywords: Electrical resistivity, Multilayer thin films, Interface, Grain boundary, Temperature coefficient of resistivity

\section{Introduction}

Multilayer films often exhibit unique mechanical and physical properties [1-4], and thus have been extensively studied. The nature of layer interfaces often plays a critical role in determining the physical properties of these multilayers. Instance $\mathrm{Cu} / \mathrm{V}$ multilayers with immiscible layer interfaces have size dependent mechanical properties, and radiation tolerance, that is a smaller individual layer thickness (h) leads to greater mechanical strength and enhanced radiation tolerance (such as lower He bubble density) [5, 6]. The immiscible layer interfaces are natural defect sinks and reduce the density of radiation induced defects substantially. It is well-known that the electrical resistivity of metallic multilayers can be tuned by layer interfaces which usually act as obstacles to charge carriers [7]. But the electrical resistivity of $\mathrm{Cu} / \mathrm{V}$ multilayer films has not been investigated to date. 
P. F. Carcia and A. Suna studied the resistivity of $\mathrm{Pd} / \mathrm{Au}$ multilayer film and they considered the interface scattering as the only factor in determining the resistivity [8]. Later studies showed that the resistivity of films is determined by the defect scattering, phonon scattering, impurity scattering, interface scattering and grain boundary (GB) scattering. The electron mean free path and temperature are important factors that affect the resistivity of in numerous metallic multilayer systems, such as $\mathrm{Nb} / \mathrm{Pb}, \mathrm{Cu} / \mathrm{Cr}, \mathrm{Cu} / \mathrm{Nb}$ and $\mathrm{Ag} / \mathrm{Cu}$ [9-12].

Several mechanisms and models have been used to interpret the electrical resistivity of metal film and metallic multilayers [13-18]. The Fuchs-Sondheimer (F-S) model was proposed to study the role of interface scattering in determining the electrical resistivity in metallic multilayer film by using original semi-classical solution [13, 14]. Then Mayadas and Shatzkes proposed a model to explain the behavior of electrical resistivity of thin films by considering the grain boundary effect (M-S model) [15]. Based on the M-S model, Dimmich took the effect of interface scattering into consideration by using boundary conditions to study the electrical resistivity of multilayer film and the expression for resistivity is given [16]:

$$
\rho=\frac{1+m \gamma}{M_{1}+m \gamma M_{2}} \rho_{0}
$$

where $\mathrm{m}$ is the ratio of one metal individual layer thickness to another metal individual layer thickness when the metallic multilayer film is composed of two metals; $\gamma$ is the ratio of the bulk resistivity $\left(\rho_{0}\right)$ of the two metals; $\mathrm{M}_{1}$ and $\mathrm{M}_{2}$ are functions of $\mathrm{k}, \mathrm{p}, 1$ and $\mathrm{R}$; $\mathrm{k}$ is the ratio of individual layer thickness (h) to intrinsic electron mean free path (l); $p$ is the reflection coefficient of specular surface; $\mathrm{R}$ is the reflection of the coefficient in the grain boundary.

Dimmich's study on the temperature coefficient of resistivity (TCR) of metallic multilayer films within the framework of Boltzmann kinetic theory shows a theoretical relationship between the resistivity and the temperature for a multilayer film [16]. TCR of the film represents the relative change in resistivity when the temperature changes, and is defined as:

$$
\alpha=\frac{1}{\rho} \frac{d \rho}{d T}
$$

where $\rho$ can be obtained from Dimmich's expression of Eq. (1).

Based on Dimmich's work, M. Fenn and co-workers studied the electrical resistivity of $\mathrm{Cu}$ and $\mathrm{Nb}$ thin films together with $\mathrm{Cu} / \mathrm{Nb}$ multilayers in terms of internal structure of $\mathrm{Cu}$ and $\mathrm{Nb}$ films and verified that Dimmich's theory provides a good description of the relationship between the resistivity and the thickness of the $\mathrm{Cu}$ and $\mathrm{Nb}$ thin films together with the individual layer thickness of the $\mathrm{Cu} / \mathrm{Nb}$ multilayer films $[17,18]$.

The F-S model and M-S model mentioned above are dedicated on studying how the interface and the grain boundary affect the resistivity of the single-layer film. For the multilayer film, both the interface scattering and GB scattering should be considered, and thus many studies described the resistivity and the TCR of multilayer film by considering the two factors. However, the effect of the proportion of the two scattering factors on the resistivity is not well considered, and thus the established mechanisms and models are needed to improve. This article systematically studies the temperature and size effect on 
the electrical resistivity of $\mathrm{Cu} / \mathrm{V}$ multilayer films by considering the proportion of the interface scattering and GB scattering.

In this paper, the interface density is defined as the ratio of total thickness to individual layer thickness (h) of the multilayer film. The interface density increases with the decrease of individual layer thickness. A new model is proposed to understand the fundamental mechanisms of the electrical resistivity as a function of individual layer thickness in metallic multilayer film by considering roles of the interface scattering, GB scattering and the inherent background scattering based on F-S model, M-S model and Dimmich's expression.

\section{Experimental}

The $\mathrm{Cu} / \mathrm{V}$ multilayer films with individual layer thickness of $2.5 \mathrm{~nm}, 5 \mathrm{~nm}, 10 \mathrm{~nm}$, $25 \mathrm{~nm}, 50 \mathrm{~nm}, 100 \mathrm{~nm}$, together with $\mathrm{Cu}$ and V single layer films, were deposited on Si (100) substrate with $\mathrm{SiO}_{2}$ layer on the top of the substrate by magnetron sputtering system at room temperature, respectively. The base pressure in the chamber was less than $5 \times$ $10^{-5} \mathrm{~Pa}$. Argon partial pressure during sputtering was $0.5 \mathrm{~Pa}$. The thickness of $\mathrm{SiO}_{2}$ layer is a few hundred nanometers. The part of substrate was covered by $\mathrm{Al}$ sheet prior to deposition, so the thickness of thin film after deposition can be obtained by measuring the step height between the region without deposition and region with the film by stylus profiling system. Then the thickness of film was divided by the deposition time to get deposition rate. The deposition rates for Cu layer and V layer are $0.39 \mathrm{~nm} / \mathrm{s}$ and $0.15 \mathrm{~nm} / \mathrm{s}$, respectively. The total thickness of multilayer film was kept at $\sim 1000 \mathrm{~nm}$ during deposition.

The electrical resistance of the multilayer film was measured by a standard four-probe method with the temperature ranging from 150 to $300 \mathrm{~K}$. The electrical resistivity can be calculated by:

$$
\rho=\mathrm{R} \frac{S}{d}
$$

where $\mathrm{R}$ is electrical resistance, $\mathrm{d}$ is the distance between two electrodes and $\mathrm{S}$ is the cross sectional area.

The microstructure of $\mathrm{Cu} / \mathrm{V}$ multilayer film was characterized by $\mathrm{X}$-ray powder diffractometer (XRD) and transmission electron microscopy (TEM). XRD was performed on PANalytical X-Pert3 Powder working at $60 \mathrm{kV}$ and $55 \mathrm{~mA}$ using $\mathrm{Cu} \mathrm{K} \alpha_{1}(\lambda=1.54056$ $\AA$ ) radiation and TEM was performed on a FEI Tecnai F30 microscope operating at 300 $\mathrm{kV}$ with a field-emission gun. The cross-sectional TEM specimens were prepared by the standard procedures consisting of slicing, grinding, and finally low energy (3.5keV) Ar ion milling and subsequent lower energy ion polishing. In detail, firstly, a sandwich structure is prepared by bonding two rectangular slices of multilayers facing each other with M-bond 610. Then the sandwich structure sample was glued vertical to the glass column and then was mechanically reduced to $25-30 \mu \mathrm{m}$ thick by grinding and polishing. After that the sample was bonded onto molybdenum ring with a diameter of $3 \mathrm{~mm}$ followed by $\mathrm{Ar}$ ion milling. In order to minimize the damage induced by ion milling, the Ar ion polishing with the energy of $1.5 \mathrm{keV}$ and the angle of $1^{\circ}$ was applied to the sample to achieve the cross-sectional TEM specimens in the final step. 


\section{Results and Discussions}

\subsection{Microstructure of $\mathrm{Cu} / \mathrm{V}$ multilayer films}

Fig. 1a and Fig. 1b are the cross-sectional TEM images and corresponding selected area diffraction $(\mathrm{SAD})$ patterns of $\mathrm{Cu} / \mathrm{V} 10 \mathrm{~nm}$ and $\mathrm{Cu} / \mathrm{V} 100 \mathrm{~nm}$ multilayer films, respectively. The layer interface of $\mathrm{Cu} / \mathrm{V}$ multilayer films is distinguishable due to their immiscibility. X-ray diffraction $\theta-2 \theta$ patterns (not shown here) indicate that the $\mathrm{Cu}$ layers in the multilayer films have a strong (111) texture, while the V layers have a (110) texture. The grain sizes of $\mathrm{Cu}$ and $\mathrm{V}$ in $\mathrm{Cu} / \mathrm{V}$ multilayer films were calculated by the Scherrer's equation based on XRD results and listed in Table 1. It shows that the grain size increases as the individual layer thickness increases. The grain sizes in the multilayer film are consistent with the measurement from the cross-sectional TEM images which exhibit columnar-grain microstructures in the multilayer.

\subsection{The temperature effect on the electrical resistivity of $\mathrm{Cu} / \mathrm{V}$ multilayer films}

The room temperature $(300 \mathrm{~K})$ electrical resistivity $(\rho)$ of bulk $\mathrm{Cu}$ and $\mathrm{V}$ is $\sim 1.7$ and $\sim 22.6 \mu \Omega \cdot \mathrm{cm}$, respectively $[19,20]$. The resistivity of $\mathrm{Cu}$ and $\mathrm{V}$ single layer film with the thickness of $1000 \mathrm{~nm}$ measured at $300 \mathrm{~K}$ is $\sim 2.15 \mu \Omega \cdot \mathrm{cm}$ and $\sim 68.75 \mu \Omega \cdot \mathrm{cm}$. The phenomenon where the resistivity of thin film is higher than that of the counterpart of the bulk has been explained by the well-known Fuchs size-effect theory in terms of the existence of the surface scattering [14].

The resistivity of a metal film can be given from the empirical Mattheissen rule as [21, 22]:

$$
\rho_{f}=\rho_{b}(T)+\rho_{d f}\left(n_{d}\right)+\rho_{s}(h)
$$

where $\rho_{b}, \rho_{d f}$ and $\rho_{s}$ are the contributions to the film resistivity caused by the phonon scattering, imperfection of the crystalline lattice, and the boundaries of the film. $\rho_{b}$ depends on the temperature and $\rho_{d f}$ depends on the defect concentration [19].

Fig. 2 shows that the resistivity for $\mathrm{Cu}$ and $\mathrm{V}$ single layer films increases when the temperature increases from $150 \mathrm{~K}$ to $300 \mathrm{~K}$. The increase of temperature results in the increase of lattice vibration amplitude and density of point defects. Thus, higher temperature leads to the smaller electron mobility and more electrons and phonons scattering, which leads to the increase of the resistivity of metallic films.

To quantitatively describe the relationship between the resistivity and the temperature, the TCR of $\mathrm{Cu}$ and V single layer film is calculated according to Eq (2) and can be extracted as follows in the current study:

$$
\alpha=\frac{1}{\rho(150 K)} \frac{\rho(300 K)-\rho(150 K)}{300 K-150 K}
$$

Eq. (1) and Eq. (2) show that the TCR is the function of t, 1, p and R. For the single layer film, the TCR can be affected by the film thickness and grain size. Some studies show that the TCR of the single metal film increases as the film thickness and the grain size increase [17]. In present results, the $\mathrm{Cu}$ and $\mathrm{V}$ single layer films with the thickness of 1000nm have TCR of $0.006 K^{-1}$ and $0.004 K^{-1}$, respectively. The TCR value of bulk $\mathrm{Cu}$ and $\mathrm{V}$ are $0.03 K^{-1}$ and $0.02 K^{-1}$ [18], which are larger than the metal films. It is mainly because that the thin film has higher concentration of scattering factors such as interface and grain boundary that are less dependent on the temperature than bulk metal [19]. These scattering 
factors such as interface scattering and impurity scattering make a greater contribution to the resistivity of the film and make the TCR of thin film smaller than the bulk metal.

Fig. 3 shows that the resistivity of the $\mathrm{Cu} / \mathrm{V}$ multilayer films with the individual layer thickness from $2.5 \mathrm{~nm}$ to $100 \mathrm{~nm}$ increases for all the samples when the temperature increases from $150 \mathrm{~K}$ to $300 \mathrm{~K}$. The increase of resistivity in $\mathrm{Cu} / \mathrm{V}$ multilayer films with the increase of the temperature is due to smaller electron mobility and more electrons scattering induced by the increase of temperature. It is also found that the resistivity of the multilayer increases as the interface density increases in the temperature range of $150 \mathrm{~K}$ to $300 \mathrm{~K}$.

Fig. 4 shows the comparison of the resistivity as a function of the individual layer thickness of $\mathrm{Cu} / \mathrm{V}$ multilayer at $150 \mathrm{~K}$ and $300 \mathrm{~K}$. It shows that the resistivity decreases when the individual layer thickness increases (interface density decreases) at the temperatures of $150 \mathrm{~K}$ and $300 \mathrm{~K}$. The interface acting as a planar defect increases the electron scattering. As a result, higher interface density results in higher resistivity. Fig. 4 also shows the TCR of the $\mathrm{Cu} / \mathrm{V}$ multilayer films and the rule-of-mixture (ROM) TCR value of $1000 \mathrm{~nm} \mathrm{Cu}$ and $\mathrm{V}$ single layer thin films obtained in the temperature range from $150 \mathrm{~K}$ to $300 \mathrm{~K}$. The TCR of $\mathrm{Cu} / \mathrm{V}$ multilayer is much smaller than that of ROM when the individual layer thickness is larger than $2.5 \mathrm{~nm}$. It could be due to that the TCR of multilayer is also related with the interface density and structure. The TCR value of multilayer is calculated from the change of resistivity of multilayer films by Eq. (5). For the multilayers, the interfaces may act as sinks to absorb some intrinsic point defects that are largely influenced by temperature, and therefore the resistivity of multilayer films will change less with the changing temperature compared with monolayer films. As a result, the TCR of $\mathrm{Cu} / \mathrm{V}$ multilayer film is smaller than ROM TCR of $\mathrm{Cu}$ and $\mathrm{V}$ single layer films. Furthermore, it can be found that TCR decreases as the individual layer thickness decreases when the individual layer thickness is larger than $2.5 \mathrm{~nm}$. It could be due to the difference of the interface density in the multilayers.

According to Eq. (4), the TCR are mainly affected by the change of $\rho_{b}$ and $\rho_{d f}$ as $\rho_{s}$ is not sensitive to the temperature. The TCR in Eq. (5) can then be written as follows:

$$
\begin{aligned}
& \text { TCR }=\frac{1}{\rho_{150 K}} \frac{\left[\rho_{b}(T)+\rho_{d f}\left(n_{d}\right)\right]_{300 K}-\left[\rho_{b}(T)+\rho_{d f}\left(n_{d}\right)\right]_{150 K}}{150 K} \\
& =\frac{1}{\rho_{150 K}} \frac{\Delta\left[\rho_{b}(T)+\rho_{d f}\left(n_{d}\right)\right]}{150 K}
\end{aligned}
$$

The interface in the multilayer has two effects on the resistivity and therefore influences TCR value. Firstly, interface absorbs phonon defects and thermal defects and higher interface density can absorb them more. The defects are dependent on the temperature. The TCR of the multilayer reduces with the increase of interface density as the resistivity changes less with the increase of temperature due to higher interface density. Secondly, the resistivity caused by interface will not change with temperature as interface is independent of temperature. The interface in the multilayer decreases the mean free path of electron and its effect on the resistivity becomes larger when its density increases. Therefore, higher interface density contributed more to the total resistivity of multilayers, 
and defects contributed less to the total resistivity of multilayers. With the increase of temperature, the changes of resistivity induced by defects will be less. Meanwhile, the multilayer with higher interface density has a larger $\rho_{150 K}$. As a result, the TCR value in multilayers reduces as the interface density increases. Thus, integrated above reasons, the multilayer with higher interface density will have smaller TCR.

The $\mathrm{Cu} / \mathrm{V}$ multilayer with the highest interface density should have the largest resistivity and smallest TCR in multilayers. However, $\mathrm{Cu} / \mathrm{V} 2.5 \mathrm{~nm}$ multilayer film has the largest TCR although it has the highest interface density. The influence of the factors on the resistivity of $\mathrm{Cu} / \mathrm{V} 2.5 \mathrm{~nm}$ multilayer could be related to the internal structure of interface and the process of the film formation. Individual layer thickness of $2.5 \mathrm{~nm}$ may be too thin to form a network structure, but a structure more like island structure. In this period of film formation, the conductive mechanism could be thermionic emission and tunnel movement. Thus the $\mathrm{Cu} / \mathrm{V} 2.5 \mathrm{~nm}$ multilayer may show greater dependence on the temperature than other multilayers.

\subsection{The size effect on electrical resistivity of $\mathrm{Cu} / \mathrm{V}$ multilayer films}

Fig. 4 shows that the resistivity of $\mathrm{Cu} / \mathrm{V}$ multilayer films decreases when the individual layer thickness increases at the temperatures $300 \mathrm{~K}$. The resistivity of the multilayer film depends on several factors. For the individual layer of multilayer film, the electrical conduction mechanisms largely depend on the microstructure of film, which varies in different stages of growth because of the kinetics and the thermodynamics of the metal atoms on the substrate surface [23]. The individual layer film has the island shaped microstructure when it starts to form, so the $\mathrm{Cu}$ and $\mathrm{V}$ individual layer with small thickness shows the non-metallic electrical property and their resistivity is much larger than the corresponding metallic film. As the individual layer thickness increases to a critical value during individual layer growth, it becomes to network structure, where the electrical conduction mechanism is changed to percolation conduction $[24,25]$. Its resistivity drops rapidly as long as the transformation of electrical property from non-metal to metal occurs. Therefore, the change rate of the resistivity becomes small after the individual layer thickness increases to the critical value where the microstructure of the individual layer film becomes continuous and complete network structure.

In general, the resistivity of the metallic film is determined by the combination of the interface scattering, defects and impurity scattering, and GB scattering [10, 11]. Considering the interface scattering only in the metallic film, Fuchs-Sondheimer (F-S) model has been proposed by using original semi-classical solution $[13,14]$. The equation of F-S model for the resistivity of metallic thin films is given as follows [14]:

$$
\rho=\rho_{0}\left[1-(3 / 2 k)(1-p) \int_{1}^{\infty}\left(\frac{1}{\varepsilon^{3}}-\frac{1}{\varepsilon^{5}}\right) \frac{1-e^{-k \varepsilon}}{1-p e^{-k \varepsilon}} d \varepsilon\right]^{-1}
$$

where $\rho_{0}, \mathrm{k}, 1$ and $\mathrm{p}$ have the same meaning as ones in Eq (1). The formula can be simplified as follows when $\mathrm{k}$ is greater than $1[19]$ :

$$
\rho=\rho_{0}\left[1+\frac{3}{8 k}(1-\mathrm{p})\right]
$$

Fuchs postulates external surface as a boundary condition and applies it to the electron-distribution function. The results show that the thickness of thin film as additional 
factor affects the total resistivity $\rho$ [13].

The resistivity of the film will decrease when the change of film's microstructure is from island to continuous network. For the continuous network metal films, the thickness of the film also influences the film resistivity such as Fuchs size effect [14]. When the thickness of film is comparable with the mean free path of the bulk metal, the surface can also impose a geometric restriction on the movement of the conduction electrons. This is so-called the Fuchs size effect. This effect induces an increase in resistivity of the metal film, however, the magnitude of the increase in resistivity decreases when the thickness of continuous network film increases. Thus the resistivity of the film decreases as the thickness of the metal film increases due to Fuchs size effect.

Besides interface scattering, another important factor that needs to be considered is grain boundary effect. For the bulk metal with grain size of micro meter, the grain size is usually much larger than the mean free path caused by other scattering matters such as phonon and impurity, so that the grain-boundary contribution to the resistivity is small and can be negligible [26]. However, for the thin films, the grain size is generally smaller than the mean free path, thus the GB scattering cannot be negligible [15]. Considering the GB scattering in the thin film, Mayadas and Shatzkes proposed a model as follows [15]:

$$
\rho=\rho_{0}\left[1+\frac{3}{2} \frac{l R}{D(1-R)}\right]
$$

where $\mathrm{D}$ is the grain size, which is a function of individual layer thickness as shown in Table 1. R (reflection coefficient of grain boundary) is achieved by the Dimmich's theory [16]. The grain boundary can prevent the electron or phonon to moving in the same direction with the same velocity and energy and thus result in smaller mean free path and smaller electron mobility. Hence the GB scattering will be a factor that increases the resistivity of the thin film. Smaller grain size results in the increase of the grain boundary per unit volume and as a result the resistivity of the thin film with smaller grain size is larger.

To study how the interface scattering and GB scattering make a contribution to the resistivity of $\mathrm{Cu} / \mathrm{V}$ multilayer, F-S model and $\mathrm{M}-\mathrm{S}$ model have been used to fit the experimental data. Some assumptions have been made during the calculation and fitting. Firstly, the $\mathrm{p}(0.5)$ is set as the same for the electron crossing both from the $\mathrm{Cu}$ layer to $\mathrm{V}$ layer and the other direction. Secondly, an average mean-free path (l) and $\mathrm{R}$ of $\mathrm{Cu}$ $1 \mu \mathrm{m}$-single layer film and V $1 \mu \mathrm{m}$-single layer film are used in the model. The mean-free path can be calculated below [10]:

$$
\mathrm{l}=\frac{h}{2 \pi} \frac{1}{e^{2} \rho_{1 \mu m}}\left(\frac{\sqrt{3} \pi}{n}\right)^{2 / 3}
$$

where e is the electron charge, $\mathrm{h}$ is Planck's constant, $\mathrm{n}$ is carrier concentration and $\rho_{1 \mu m}$ is the resistivity of $\mathrm{Cu}$ or $\mathrm{V} 1 \mu \mathrm{m}$-single layer film. The average 1 is about $9 \mathrm{~nm}$ at $300 \mathrm{k}$. Thirdly, other background scattering contributions such as phonon and impurity scattering are included in the bulk resistivities $\left(\rho_{0}\right) . \rho_{0}$ is calculated from the bulk resistivity of $\mathrm{Cu}$ $\left(\rho_{0 C u}\right)$ and $\mathrm{V}\left(\rho_{0 V}\right)$ with the law of resistance in parallel or in series.

Fig. 5a shows the schematic diagram of the circuit in the multilayer film to modulate the measurement of the resistivity by four-probe method. The 4 points in the Fig. 5a 
represent the four electrodes. The fixed current flows from point 1 to point 4 through the whole multilayer film. The resistance is obtained through four-probe method by measuring the voltage between point 2 and point 3 . Then the resistivity is obtained by calculating through Eq (3).

When considering the interface scattering in F-S model, each bilayer of $\mathrm{Cu}$ and $\mathrm{V}$ is considered as one resistor outlined by the red dashed box shown in Fig. 5a. In this case, the $\mathrm{Cu} / \mathrm{V}$ multilayer film is composed of the same resistors acting in parallel shown in Fig. 5 b. The intrinsic resistivity of each resistor $\left(\rho_{0}\right)$ can be calculated from $\rho_{0 \mathrm{Cu}}$ and $\rho_{0 \mathrm{~V}}$ with the law of resistance in parallel $\left(\rho_{\text {op }}\right)$ and the resistivity of each resistor $\left(\rho_{\text {in }}\right)$ can be obtained by Eq. (8). Thus the resistivity considering the interface scattering $(\rho)$ can be calculated from $\rho_{\text {in }}$ with the law of resistance in parallel.

In the $\mathrm{M}-\mathrm{S}$ model, when considering the $\mathrm{GB}$ scattering, the column grains in $\mathrm{Cu} / \mathrm{V}$ multilayers are regarded as parallel block perpendicular to the interface outlined by the blue dotted box shown in Fig. 5a. The $\mathrm{Cu} / \mathrm{V}$ multilayer film is composed of the same resistors acting in series as shown in Fig. 5c. Thus the intrinsic resistivity of each resistor $\left(\rho_{0}\right)$ is calculated from $\rho_{0 \mathrm{Cu}}$ and $\rho_{0 \mathrm{~V}}$ with the law of resistance in series $\left(\rho_{0 \mathrm{~s}}\right)$. The resistivity of each resistor $\left(\rho_{g}\right)$ can be obtained by Eq. (9). Thus the resistivity considering the GB scattering $(\rho)$ can be calculated from $\rho_{g}$ with the law of resistance in series.

With the assumptions above, F-S model (red curve) and M-S model (blue curve) are used to fit the resistivity of $\mathrm{Cu} / \mathrm{V}$ multilayer films measured at $300 \mathrm{~K}$ shown in Fig. 6. People usually pointed out that interface scattering as a reason leads to the rapid increase of resistivity. According to the data, it can be found the gradual increase in $\rho$ with decreasing $\mathrm{h}$ from 100 to $25 \mathrm{~nm}$, and rapid increase in $\rho$ with decreasing $\mathrm{h}$, for $\mathrm{h}<25 \mathrm{~nm}$. So interface scattering may be thought to make a more important role at low $\mathrm{h}$ in the experiment for $\mathrm{Cu} / \mathrm{V}$ multilayer film. However, Fig. 6 shows that the $\rho$ estimated by the F-S model is smaller than the experimental data. This indicates that considering the effect of interface scattering only on the resistivity of $\mathrm{Cu} / \mathrm{V}$ multilayer is not sufficient to predict the resistivity of $\mathrm{Cu} / \mathrm{V}$ multilayer film.

Fig. 6 also shows that the M-S model is coincidence with the data when the individual layer thickness is larger, inferring that GB scattering is the main factor in determining the resistivity in this region. Table 1 shows that the ratio of grain size to individual layer thickness is getting smaller as the individual layer thickness becomes larger. In this region ( $\mathrm{h}>25 \mathrm{~nm}$ ), both the individual layer thickness and the grain size is larger than the mean free path of $\mathrm{Cu}(\sim 17 \mathrm{~nm})$ or $\mathrm{V}$ films $(\sim 0.46 \mathrm{~nm})$, however, the grain size is much closer to mean free path and thus GB scattering plays more important role in determining the resistivity than the interface scattering. Hence we may imply that the main contributor to the resistivity is the GB scattering when individual layer thickness is larger. The resistivity will slowly rise resulting from that the grain size is getting small as the individual layer thickness decreases. However, when the individual layer thickness decreases to a critical size and much closer to the mean free path, interface scattering becomes the main contributor to the resistivity. Thus the resistivity will grow rapidly as the interface density in the film increases in this region.

To quantitatively explain the experimental data with the interface scattering and GB scattering, the F-S model and M-S model are combined to develop a new model. In Eq. (8), 
the simplified formula of the Fuchs theory is used when considering the interface scattering, which has a limiting condition of $\mathrm{k}$ greater than $1(\mathrm{~h}>9 \mathrm{~nm})$. When $\mathrm{h}$ is too small to satisfy this requirement, this model will lose efficacy. So the factor $e^{-h / l}$ is added in the model as shown in Eq. (11). Interface can act as a restriction on the movement of the conduction electrons. Smaller individual layer thickness provides higher interface density and introduces more electrons scattering in the multilayer film. This factor considers the influence of the individual layer thickness on the mean free path, which makes the model more applicable to the situation where $\mathrm{k}$ is small. Furthermore, the proportion of the two scattering mechanisms should not be a constant value when the individual layer thickness varies from 2.5 to $100 \mathrm{~nm}$. To take interface scattering into account, a proportion factor $\mathrm{e}^{-\mathrm{h}}$ is used. This factor increases as $\mathrm{h}$ decreases. It can well reflect that the interface scattering plays the main role in affecting the resistivity at low $\mathrm{h}$ and the resistivity will grow rapidly as $h$ increases. When $h$ is getting larger, the factor becomes small. Then the GB scattering will take over the interface scattering to play dominant roles.

The modified model is written as

$$
\rho=\rho_{0 p} e^{-h} \times\left(1+\frac{3 l}{8 h}(1-\mathrm{p}) e^{-\frac{h}{l}}\right)+\rho_{0 s}\left(1+\frac{3}{2} \frac{l R}{D(1-R)}\right)
$$

The new model, shown as red solid line in Fig. 7, appears to fit the size dependent resistivity of $\mathrm{Cu} / \mathrm{V}$ multilayer films better than the two other (F-S and $\mathrm{M}-\mathrm{S}$ ) models. The improvement of the new model arises from the consideration of both the interface scattering and GB scattering effect, and size dependent proportion factors. The disparity between the model and experimental data is probably due to the usage of an average mean-free path (1) and $\mathrm{R}$ for $\mathrm{Cu}$ and $\mathrm{V}$ in the model.

Thus to correct our model, we calculated the interface scattering of $\mathrm{Cu}, \mathrm{V}$ with different $\mathrm{R}$ and 1 , respectively.

When considering the interface scattering, the mean free path of $\mathrm{Cu}$ layers $\left(l_{1}\right)$ and $\mathrm{V}$ layers $\left(l_{2}\right)$ are $17.34 \mathrm{~nm}$ and $0.46 \mathrm{~nm}$, respectively. Each single layer of $\mathrm{Cu}$ or $\mathrm{V}$ is regarded as one resistor replacing the resistor outlined by the red dashed boxes shown in Fig. 5a.

When considering the GB scattering, the reflection coefficient of grain boundary of $\mathrm{Cu}$ $\left(R_{1}\right)$ and $\mathrm{V}\left(R_{2}\right)$ are 0.24 and 0.15 , respectively.

With the correction above, the final model can be written like this:

$$
\begin{gathered}
\rho=\rho_{0 p}+\frac{\rho_{0 p}}{2}\left[\frac{3 l_{1}}{8 h}(1-\mathrm{p}) e^{-\frac{h}{l_{1}}}+\frac{3 l_{2}}{8 h}(1-\mathrm{p}) e^{-\frac{h}{l_{2}}}\right] e^{-h} \\
+\frac{\rho_{0 s}}{2}\left[\frac{3}{2} \frac{l_{1} R_{1}}{D_{1}\left(1-R_{1}\right)}+\frac{3}{2} \frac{l_{2} R_{2}}{D_{2}\left(1-R_{2}\right)}\right]
\end{gathered}
$$

In this way, the new model can be achieved as shown in Fig. 7 (blue curve). It seems to fit well with the experimental data in $300 \mathrm{~K}$. This new model can clearly show the relationship among the resistivity, the individual layer thickness of the $\mathrm{Cu} / \mathrm{V}$ multilayer film, and two main factors who determine the resistivity of the multilayer. The size effects on resistivity of $\mathrm{Cu} / \mathrm{Cr}$ multilayer films by considering the interface scattering and $\mathrm{GB}$ scattering were investigated as well in previous study where the resistivity of $\mathrm{Cu} / \mathrm{Cr}$ multilayer films can be described by a function of intrinsic resistivity of $\mathrm{Cu}$ and $\mathrm{Cr}$ [10]. 
The function is calculated by solving one dimensional version of the Boltzmann transport equation. The intrinsic layer resistivities are those of the $\mathrm{Cu}$ and $\mathrm{Cr} 1 \mu \mathrm{m}$ single layer films, which consider all the scattering factors of resistivity such as interface scattering and GB scattering. The estimated resistivity obtained by their method is smaller than the data at all values of individual layer thickness as the intrinsic resistivity of the copper layers also decreases with decreasing bilayer period.

For the model in this study, the background scattering factors such as phonon and impurity scattering are considered in the $\rho_{0}$. The interface scattering and GB scattering are considered additionally by using the F-S model and M-S model. Thus the model can explain the contribution of the two factors more visually. The modifications in the model consider the changeful proportion of the two scattering mechanisms when determining the resistivity of $\mathrm{Cu} / \mathrm{V}$ multilayers and considering the different structures of $\mathrm{Cu}$ and $\mathrm{V}$ layers. The result shows that this model fits well with the experimental data at $300 \mathrm{~K}$.

\subsection{The Hall-Petch behavior on electrical resistivity of $\mathrm{Cu} / \mathrm{V}$ multilayer film}

The Hall-Petch scaling law describes the general relationship between the yield stress (and other mechanical properties) and grain size. Hall-Petch equation also has been found to apply not only to grain boundaries but to other kinds of boundaries [27-29]. Later studies showed that Hall-Petch equation well described the relationship between the yield strength and the individual layer thickness of the multilayer films [30, 31]. In metallic multilayer, the yield strength is proportional to $\mathrm{h}^{-1 / 2}$ as the dislocation pile-ups at layer interfaces [32]. It is interesting that a relationship following Hall-Petch law has been found between the resistivity and the individual layer thickness of the $\mathrm{Cu} / \mathrm{V}$ multilayer film.

Fig. 8 shows the resistivity as a function of the $h^{-1 / 2}$ in $\mathrm{Cu} / \mathrm{V}$ multilayer at $300 \mathrm{~K}$, where $\mathrm{h}$ is the individual layer thickness. A linear fit to the data is found to be consistent with the Hall-Petch behavior when the individual layer thickness is not smaller than $5 \mathrm{~nm}$ :

$$
\rho=\rho_{0 p}+b h^{-1 / 2}
$$

where $\rho_{0 p}$ is the intercept and $b$ is the slope. $\rho_{0 p}$ is $1.58 \mu \Omega \cdot \mathrm{cm}$ and is the intrinsic resistivity calculated from $\rho_{0 \mathrm{Cu}}$ and $\rho_{\mathrm{OV}}$ with the law of resistance in parallel. This indicates that the resistivity of the $\mathrm{Cu} / \mathrm{V}$ multilayer film is a parallel value of the bulk resistivity of $\mathrm{Cu}$ and $\mathrm{V}$ when the individual layer thickness is infinite. The slope (b) determines the rate of resistivity change with the change of individual layer thickness. The resistivity of multilayer is mainly depended on the interface scattering and the GB scattering. Both the individual interface thickness and the grain size affect the resistivity of $\mathrm{Cu} / \mathrm{V}$ multilayer film, and they correlate with each other. Table 1 shows that the grain size decreases as the individual layer thickness decreases. Therefore, in the Hall-Petch scaling law, individual layer thickness is used as variable to describe the resistivity of $\mathrm{Cu} / \mathrm{V}$ multilayer film.

The scattering probability of the electron in the multilayer will increase and the mean free path of the electron will decrease when the individual layer thickness and grain size decrease. The resistivity will increase as the individual layer thickness decrease and the changing rate can be reflected by the slope in Eq. (11). The effect of monolayer and grain boundary on changing the resistivity can be described by using the reflection coefficient of 
boundary. Thus, the slope composed of the reflection coefficient is proposed to describe the changing rate of resistivity and is written as follows:

$$
b=R_{1} D_{f C u}+R_{2} D_{f V}
$$

where $R_{1}$ with the value of 0.24 and $R_{2}$ with the value of 0.15 are the reflection coefficient of grain boundary of $\mathrm{Cu}$ and $\mathrm{V} ; D_{f C u}$ and $D_{f V}$ are the grain size of the $\mathrm{Cu}$ and $\mathrm{V}$ single-layer. When the thickness of the single-layer is $1 \mu \mathrm{m}, D_{f C u}$ is $77 \mathrm{~nm}$ and $D_{f V}$ is $18 \mathrm{~nm}$. Taking the values provided for $R_{1}, R_{2}, D_{f C u}$ and $D_{f V}, \mathrm{~b}$ obtains the value of 39 , which is the same as the one deducted from the Hall-Petch equations regarding the resistivity of $\mathrm{Cu} / \mathrm{V}$ multilayer film.

Fig. 8 also shows that the experimental plot of $\mathrm{Cu} / \mathrm{V} 2.5 \mathrm{~nm}$ multilayer is not coincident with the Hall-Petch behavior as its microstructure is different from others. The interface of $\mathrm{Cu} / \mathrm{V} 2.5 \mathrm{~nm}$ multilayer film is no longer smooth because of the discontinuity of each monolayer film. As a result, the Hall-Petch behavior will not be applicable in this area.

Eq. (13) is an empirical formula which uses the individual layer thickness to describe resistivity of $\mathrm{Cu} / \mathrm{V}$ multilayer film based on the Hall-Petch equation. It visually expresses the changing trend of the resistivity affected by the boundary (individual layer thickness or grain size) in the multilayer film. However, the resistivity of multilayer is affected by several scattering factors. Eq.(12) is the better choice to explain and predict resistivity of $\mathrm{Cu} / \mathrm{V}$ multilayer film as a function of individual layer thickness in principle at $300 \mathrm{~K}$.

\section{Summary and conclusions}

The resistivity of $\mathrm{Cu} / \mathrm{V}$ multilayer films with different individual layer thicknesses has been measured under different temperatures. The results show that the resistivity of the $\mathrm{Cu} / \mathrm{V}$ multilayer film increases with the increase of the temperature for all the samples. The TCR value of multilayer is determined by film thickness, grain size and interface structure. A new model has been proposed to describe the relationship between the resistivity and the individual layer thickness at $300 \mathrm{~K}$. This new model not only considers the interface scattering and the GB scattering, but also involves influence of the individual layer thickness on the mean free path, the proportion of the two scattering mechanisms, mean free path and reflection coefficient of individual layer of $\mathrm{Cu}$ and $\mathrm{V}$. It reflects the contributions of factors in determining the resistivity of $\mathrm{Cu} / \mathrm{V}$ multilayer films, which may provide the base on the fundamental understanding of the relationships between microstructure and resistivity of multilayer films.

\section{Acknowledge}

This work was supported by ITER special funding (Award number: 2015GB121004) from Ministry of Science and Technology, and by National Science Foundation of China with Award Number of 11375018 and 11528508. E.F. appreciates the support from The Recruitment Program of Global Youth Experts in China, and the Instrumental Analysis Fund of Peking University.

\section{Reference}

[1] I. K. Schuller, New class of layered materials, Phys. Rev. Lett. 44 (1980) 1597-1600. 
[2] M.R. Khan, P. Roach, I. K. Schuller, Magnetic properties of sputtered multilayers of Mo/Ni, Thin Solid Films. 122 (1984) 183-189.

[3] S. M. Durbin, J. E. Cunningham, M. E. Mochel, C. P. Flynn, Nb-Ta metal superlattices, J. Phys. F: Met. Phys. 11 (1981) 223-226.

[4] T. R. Werner, I. Banerjee, Q. S. Yang, C. M. Falco, I. K.Schuller, Localization in a three-dimensional metal, Phys. Rev. B 26 (1982) 2224-2226.

[5] E.G. Fu, Nan Li, A. Misra, R.G. Hoagland, H. Wang, X. zhang, Mechanical properties of sputtered $\mathrm{Cu} / \mathrm{V}$ and Al/Nb multilayer films, Mater. Sci. Eng., A 493 (2008) 283-287.

[6] E. G. Fu, A. Misra, H. Wang, L. Shao, X. Zhang, Interface enabled defects reduction in helium ion irradiated Cu/V nanolayers, J. Nucl. Mater 407 (2010) 178-188.

[7] M. Gurvitch, Resistivities and mean free paths in individual layers of a metallic multilayered structure, Phys. Rev. B. 34(1986) 540-546.

[8] P. F. Carcia1, A. Suna, Properties of Pd/Au thin film layered structures, J. Appl. Phys. 54, (1983) 2000-2005.

[9] A.Wawro, W. Maj, J. Majewski, Resistivity and electron mean free path of $\mathrm{NiPb}$ modulated films, Superlattices. Microstruct. 10(1991) 385-388.

[10] A. Misra, M.F. Hundley, D. Hristova, H. Kung, T.E. Mitchell, M. Nastasi, J.D. Embury, Electrical resistivity of sputtered $\mathrm{Cu} / \mathrm{Cr}$ multilayered thin films, J. Appl. Phys. 85 (1999) 302-309.

[11] A.L. Lima, X. Zhang, A. Misra, C.H. Booth, E.D. Bauer, M.F. Hundley, Length scale effects on the electronic transport properties of nanometric $\mathrm{Cu} / \mathrm{Nb}$ multilayers, Thin Solid Films. 515(2007) 3574-3579.

[12] M.Z. Wei, L.J. Xu, J. Shi, G.J. Pan, Z.H. Cao, X.K. Meng, Achieving high strength and high electrical conductivity in $\mathrm{Ag} / \mathrm{Cu}$ multilayers, Appl. Phys. Lett. 106 (2015) 011604.

[13] K. Fuchs, The conductivity of thin metallic films according to the electron theory of metals, Proc. Cambridge Philos. Soc. 34 (1938) 100-108.

[14] E.H. Sondheimer, The mean free path of electrons in metals, Advan. Phys. 1 (1952) $1-42$.

[15] A. F. Mayadas, M. Shatzkes, Electrical-resistivity model for polycrystalline films: the case of arbitrary reflection at external surface, Phys. Rev. B. 1 (1970) 1382-1389.

[16] R. Dimmich, Electronic transport properties of metallic multi-layer films, J. Phys. F: Met. Phys 15 (1985) 2477-2487.

[17] M. Fenn, G. Akuetey, P.E. Donovan, Electrical resistivity of $\mathrm{Cu}$ and $\mathrm{Nb}$ thin films, J.Phys.:Condens. Matter 10 (1998) 1707-1720.

[18] M. Fenn, A.K. Petford-Long, P.E. Donovan, Electrical resistivity of $\mathrm{Cu}$ and $\mathrm{Nb}$ thin films and multilayers, J Magn Magn Mater 198-199 (1999) 231-232.

[19] G.P. Zhigal'skii, B.K. Jones, The physical properties of thin metal films, First ed., Taylor \& Francis, London and New York, 2003.

[20] P.D. Desai, H.M. James, C.Y. Ho, Electrical resistivity of vanadium and zirconium, J. Phys. Chem. Ref. Data. 13 (1984) 1097-1130.

[21] C. Kittel, Introduction to solid state physics, Seventh ed., John Wiley \& Sons, Inc, New York, 1996.

[22] K.L. Chopra, Thin film phenomena, First ed., McGraw-Hill, New York, 1969. 
[23] M. Ohring, The materials science of thin films, Academic, New York, 1991.

[24] G. Jeffers, M.A. Dubson, P.M. Duxbury, Island-to-percolation transition during growth of metal films, J. Appl. Phys. 75 (1994) 5016-5020.

[25] A. Bunde, Fractals and disordered systems, Springer-Verlag, Berlin, 1991.

[26] J. M. Ziman, Electrons and Phonons, First ed., clarendon press, Great Britain, 1962.

[27] E.O. Hall, The deformation and ageing of mild steel: III discussion of results, Proc. Phys. Soc. B 643 (1951) 747-753.

[28] N.J. Petch, The cleavage strength of polycristals, J. Iron Steel Inst. 173 (1953) 25-28.

[29] N. Hansen, B. Ralph, The strain and grain size dependence of the flow stress of copper, Acta Matall. 30 (1982) 411-417.

[30] A. Misra, J.P. Hirth, R.G. Hoagland, Length-scale-dependent deformation mechanisms in incoheren metallic multilayered composites, Acta Mater. 53 (2005) 4817-4824.

[31] L.H. Friedman, D.C. Chrzan, Scaling theory of the Hall-Petch relation for multilayers, Phys. Rev. Lett. 81 (1998) 2715-2718.

[32] A. Misra, M. Verdier, H. Kung, J.D. Embuty, J.P. Hirth, Deformation mechanism maps for polycrystalline metallic multiplayers, Scripta Mater. 41 (1999) 973-979. 

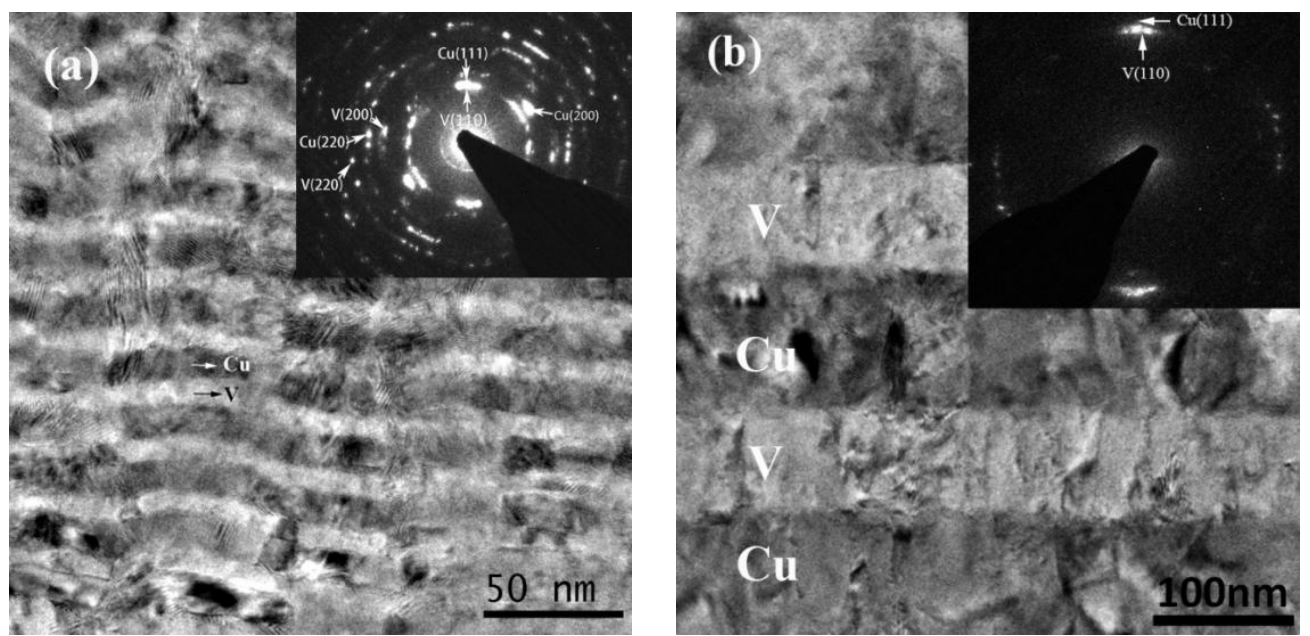

Fig.1. Cross-sectional TEM images of as-deposited (a) $\mathrm{Cu} / \mathrm{V} 10 \mathrm{~nm}$ and (b) $\mathrm{Cu} / \mathrm{V} 100 \mathrm{~nm}$ multilayer films. 

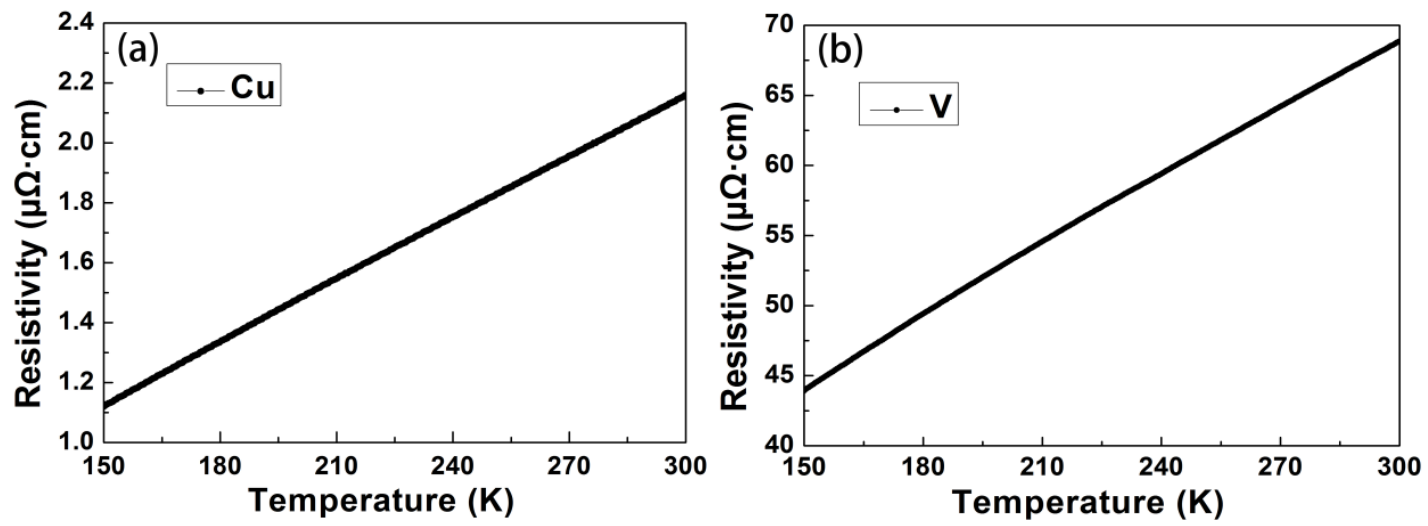

Fig.2. Temperature dependence of resistivity for single layer films of (a) $\mathrm{Cu}$, and (b) V from $150 \mathrm{~K}$ to $300 \mathrm{~K}$. 


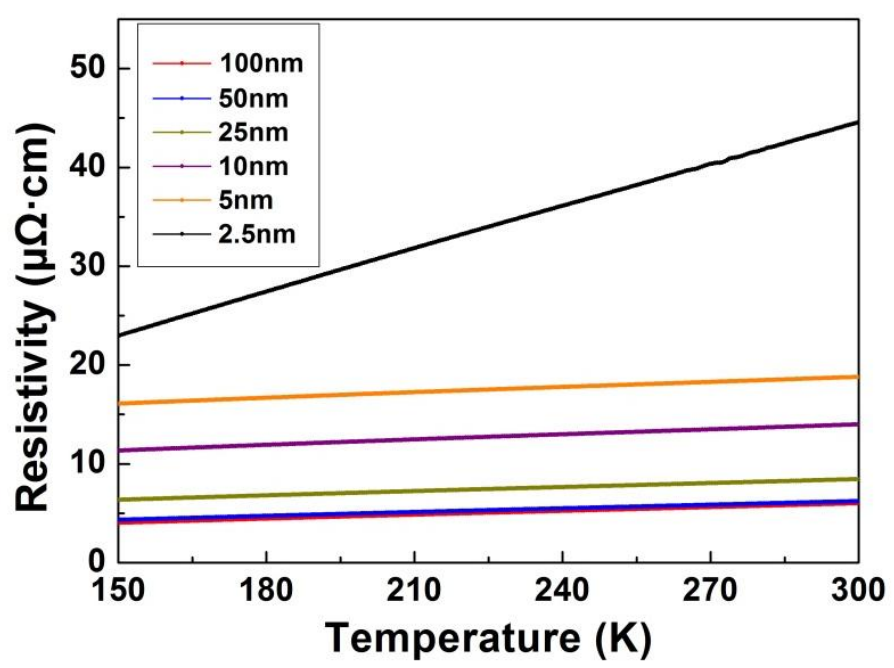

Fig.3. Temperature dependence of resistivity for $\mathrm{Cu} / \mathrm{V}$ multilayers from $150 \mathrm{~K}$ to $300 \mathrm{~K}$. 


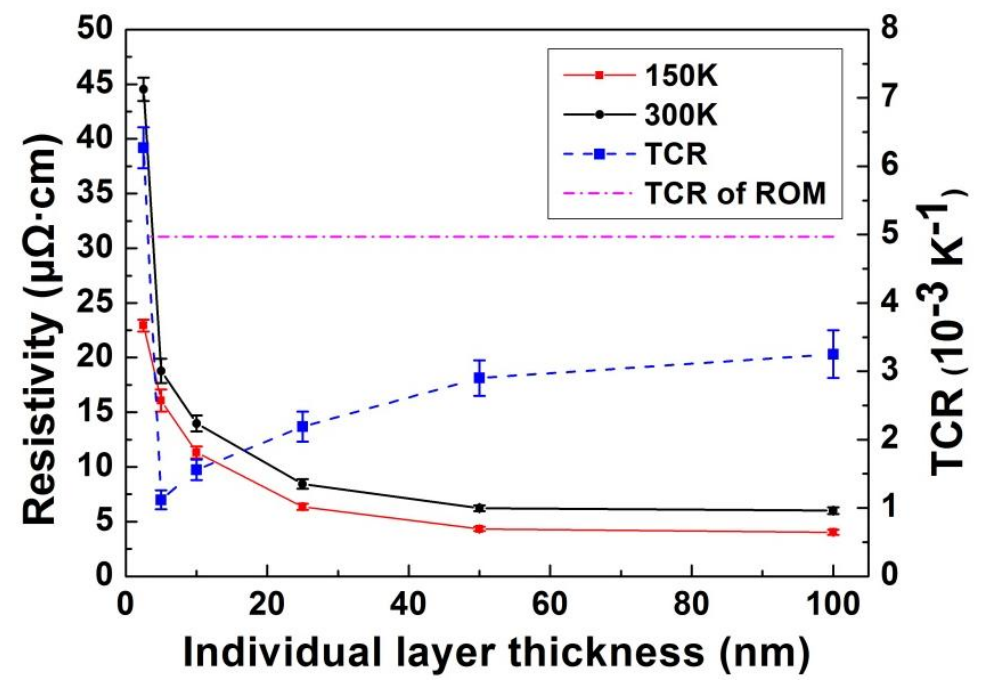

Fig.4. Comparison of resistivity as a function of individual layer thickness for multilayer films at $150 \mathrm{~K}$ and $300 \mathrm{~K}$; the TCR of multilayer and rule of mixture TCR value from $150 \mathrm{~K}$ to $300 \mathrm{~K}$ are also shown. 


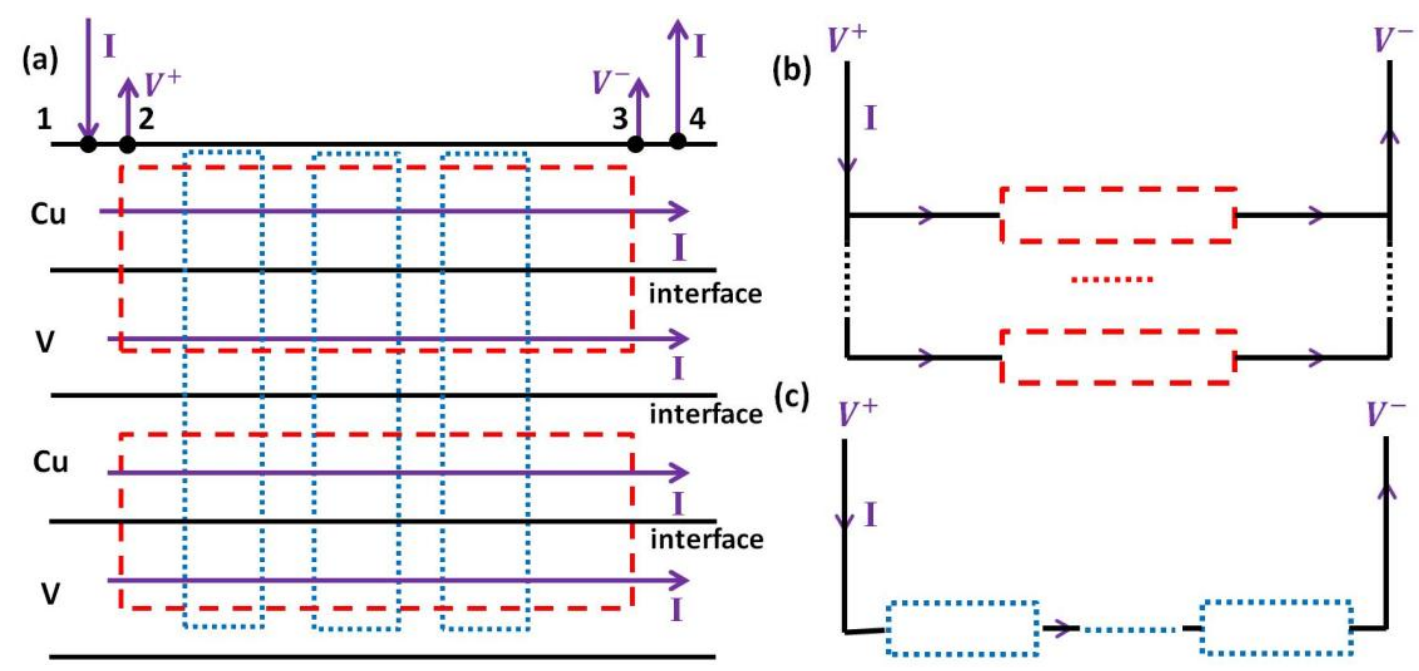

Fig.5. Schematic diagrams of (a) the circuit in the multilayer, (b) the resistance in parallel, and (c) the resistance in series when modulating the measurement of the resistivity by four-probe method. 


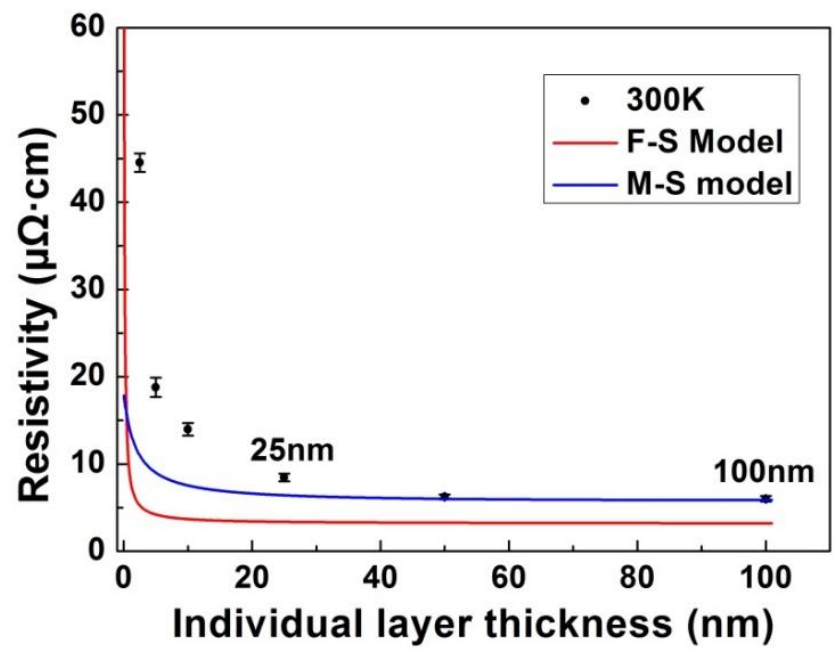

Fig.6. Comparison of the resistivity of $\mathrm{Cu} / \mathrm{V}$ multilayers measured and modulated in terms of F-S model and M-S model, respectively. 


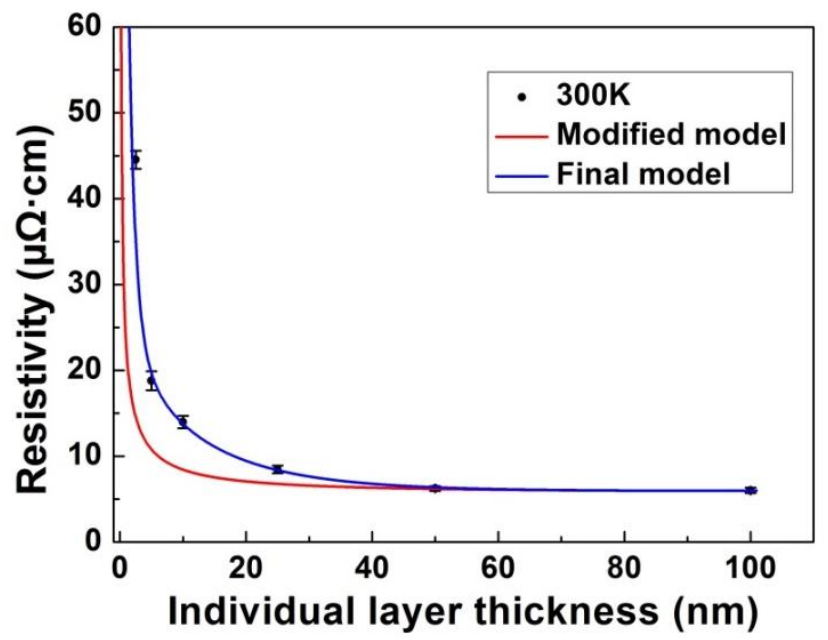

Fig.7. Comparison of the resistivity of $\mathrm{Cu} / \mathrm{V}$ multilayers measured and modulated in terms of different models we modified. 


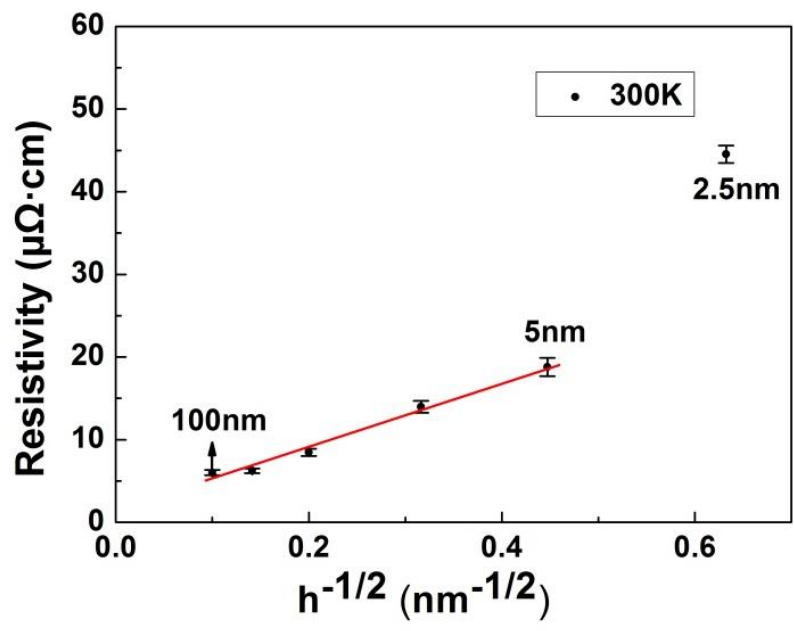

Fig.8. The resistivity vs. $\mathrm{h}^{-0.5}$ plot for $\mathrm{Cu} / \mathrm{V}$ multilayer film measured at $300 \mathrm{~K}$. 


\section{The electrical properties of $\mathrm{Cu} / \mathrm{V}$ multilayer films and its mechanism}

Table 1.

Grain size for the copper $(\mathrm{Cu})$ and vanadium $(\mathrm{V})$ layers is estimated by Scherrer's equation based on $\mathrm{XRD}$ measurement in $\mathrm{Cu} / \mathrm{V}$ multilayer films.

\begin{tabular}{lccccccc}
\hline $\begin{array}{l}\text { Individual layer thickness, } \\
\mathrm{h}(\mathrm{nm})\end{array}$ & 2.5 & 5 & 10 & 25 & 50 & 100 \\
\hline \multirow{2}{*}{ Grain size $(\mathrm{nm})$} & $\mathrm{Cu}$ & $3.3 \pm 0.1$ & $5.3 \pm 0.1$ & $12.4 \pm 0.1$ & $20.8 \pm 0.1$ & $34.6 \pm 0.1$ & $43.7 \pm 0.2$ \\
& $\mathrm{~V}$ & $3.3 \pm 0.1$ & $5.3 \pm 0.1$ & $6.3 \pm 0.1$ & $14.1 \pm 0.1$ & $24.3 \pm 0.1$ & $27.3 \pm 0.1$ \\
\hline
\end{tabular}



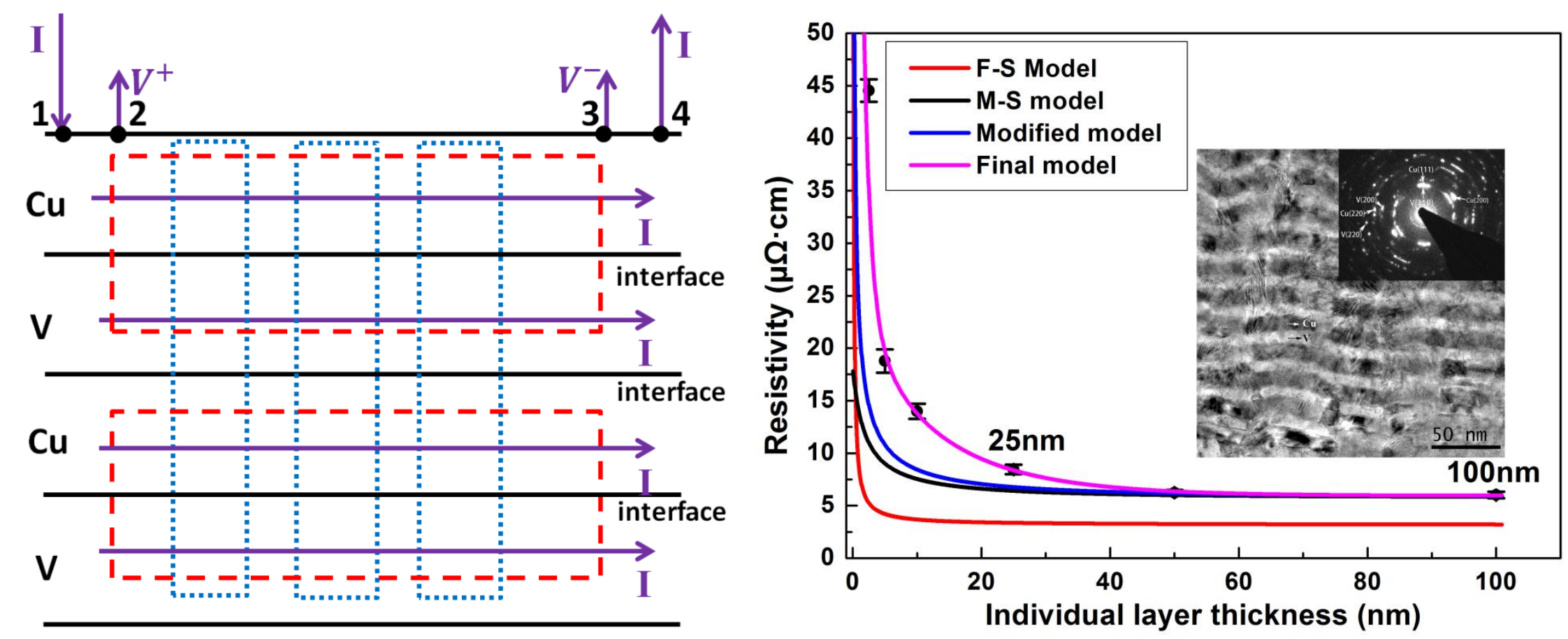